\title{
Overview on Latest Gesture Controlled Systems for Robotic Arm
}

\author{
Akash Ugale \\ Dept. of Electronics and Telecommunication Engg \\ K.K.Wagh Institute of Engineering Education \& \\ Research, Nasik 422003, INDIA
}

\author{
D M Chandwadkar, $\mathrm{PhD}$ \\ Dept. of Electronics and Telecommunication Engg \\ K.K.Wagh Institute of Engineering Education \& \\ Research, Nasik 422003, INDIA
}

\begin{abstract}
Advanced robotics is the future of technology. In the paramount ways that it can be implemented, there are some devices that can make their use very simple and effective. This paper presents one such device, that is, the leap motion technology and its implementation in a new age robotic arm. The robotic arms developed till now, either need a digital glove or a joystick to be operated. In other words, some kind of extra machinery or devices is needed to make a robotic arm work. This paper defines a robotic arm that needs no such extra help from external devices and runs solely on human command. The only human effort or manual intervention required in this would be to just make a gesture and see task getting done by the robotic arm. This arm can assist a lot in search and rescue operations. In the tough world like ours, where it is not possible for humans to reach everywhere, especially in the case of natural disasters, technology like this can come to a great aid.
\end{abstract}

\section{Keywords}

Arduino, Eclipse, Gesture Recognition, Robotic Arm, Leap Motion Technology.

\section{INTRODUCTION}

The interfacing between Man and machine is the beginning of a new era. Humans and machines no longer run parallel to each other, but instead, they go hand in hand. This new technology is helping to improve lifestyles. This motivates the creation of a better technology for tomorrow. This is called, the digital circle. The technology used in robotics earlier was of a joystick, then came the touchscreen, and now it is the advent of gestures.

There are a lot of sensors and technologies present today which detect motion and gestures. But the accuracy and speed of these sensors are not much [2][3]. The main advantage of using LEAP motion sensor is that, it is very accurate and very fast. It is even fast enough to detect handwriting. LEAP controller was initiated in 2010 , by the LEAP MOTION Company [5]. It was first implemented in 2013. Since then, the response for this sensor has been tremendous as it provides a wide arena of implementation. Many research projects are still going on for this, as people are trying to find new ways to explore its advantages.

This paper introduces a new technology to track and control the robotic arm for different activities. By moving our hand in any direction the motion can be controlled. This paper is very important since it gives an overview of various gesture controlled systems and also leap motion technology for controlling a robotic arm.

\section{AN OVERVIEW ON GESTURE CONTROLLED SYSTEMS}

A Tremendous amount of work has been already done on robotic arms and their implementations [1][2]. Such technologies can be very helpful in day to day lives, doing search and rescue operations, assisting the elderly and differently abled, performing surgeries, etc. From their help, human error can be reduced, as well as the quality of living can be improved.

The current project made on this LEAP motion is related to recognizing sign language [4]. In this project, users were able to identify 26 alphabets of the English sign language [4]. The current trend for learning sign language is via an instructor or through typed instructions. But, after this technology, the delay for learning the language can be minimized. There will be no more wait for further instructions. This can reduce the learning time and greatly aid the differently abled.

Other areas of interest are also being explored for this, like, military, medical, IT, etc. This project to primarily focuses on IT and search and rescue operations.

There are basically 2 ways to use robotics:

\section{1. contact type \\ 2. non-contact type}

The first type contains data gloves, exoskeleton, and electromagnetic tracking system. Whereas another type contains vision based system, speech recognition, camera based etc [3].For achieving this, LEAP motion sensor, robotic arm, eclipse IDE(For programming), and Arduino Module(For interfacing) are used.

Author Ganesh Choudhary proposes a way to achieve Human Computer Interface entirely in the electronic way (without mechanical sensors). To achieve the preceding idea, the image processing technique is used and for capturing motion, a web camera is used. Which detects the vital features of hand: fingers by computational geometry calculation enabling realtime interaction between hand gestures and Robot [6].

Gyro-Accelerometer based Robotic Arm using AVR Microcontroller by authors Bhuyan and Mallick is a module for improving the stability and to detect the rotational gesture of human arm the combination of gyro-meter and accelerometer are used. The 6DOF arm additionally has the capability to grab the object. For this gyro-meter gives gesture orientation data for determining dynamic gesture behavior. To evaluate the entire gesture data an artificial algorithm is used which helps to train the robotic arm [8].

Authors Biswas and Basu present their experience of using a Kinect ${ }^{\circledR}$ depth camera for recognition of some common 
gestures. Kinect depth sensor consists of an infrared laser projector combined with a monochrome CMOS sensor, which captures video data in 3D under any ambient light conditions. It generates a depth image of the subject in the plane towards the camera. For a generation of the depth profile of a subject and as a background removal the depth image is used. The difference between subsequent frames gives the motion profile of the subject and is used for recognition of gestures. In this paper, author calculates the result for 8 gestures [7].

American Sign Language Recognition Using Leap Motion Sensor module proposed by Chuan and Ragina. classifies the 26 letters of the English alphabet in American Sign Language using the derived features from the sensory data the support vector machine is used. Using movement of fingers they assign individual gesture for each alphabet. Also, they additionally introduced the parameter setting in machine learning methods and accurateness of the specific alphabet letter [4]..

\section{AN OVERVIEW ON GESTURE CONTROLLED ROBOTIC ARM USING LEAP MOTION TECHNOLOGY}

In the field of hand gesture controlled human-computer interface, the Leap Motion Controller can be considered nothing less than a breakthrough. This controller introduces a new gesture and position tracking system with pin-point accuracy. The controller operation is based on infrared optics and cameras. It can track all 10 of the human fingers simultaneously. As stated by the manufacturer, the accuracy in the detection of each fingertip position is approximately $0.01 \mathrm{~mm}$, with a frame rate of up to $300 \mathrm{fps}$. The leap motion sensor is shown in Fig.1.

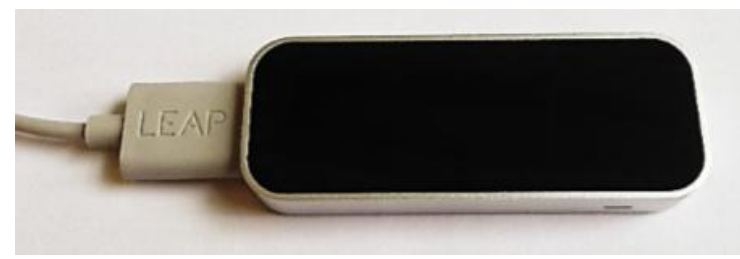

Fig.1 Leap Motion Sensor

The controller is considered to be an optical tracking system based on stereo vision. It is a compact and affordable commercialized sensor for hand and finger movements in 3D space. It has a surface area of $24 \mathrm{~cm}^{2}$, which includes three IR (Infrared Light) emitters and two IR cameras. The field view of the controller is up to $150^{\circ}$, which gives the user the opportunity to move his hand in $3 \mathrm{D}$, just like in real world. The 3D Representation is shown in Fig 2.

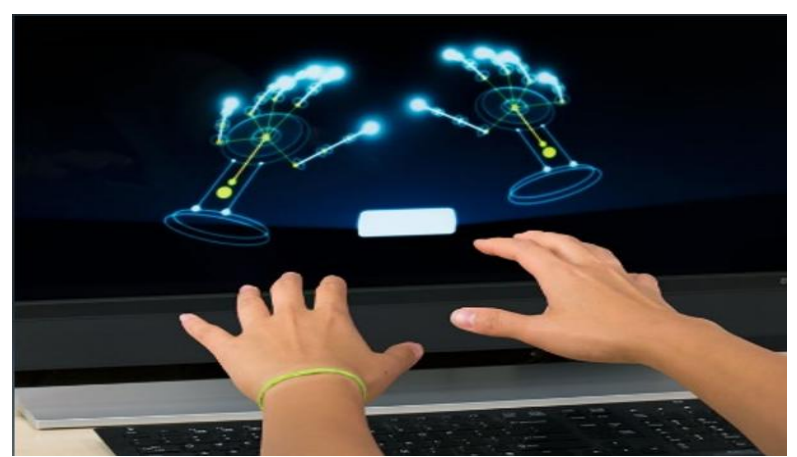

Fig. 2 3D Skeleton
The Software Development Kit (SDK) provides the information concerned with Cartesian space of predefined objects such as the finger tips, pen tip, hand palm position, etc. Along With, information dealing with the rotations of the hand such as Roll, Pitch, and Yaw are accessible as well. The Entire delivered positions are relative to the Leap Motion Controller's center point, which lies between the two IR cameras, just on top of the second IR emitter. It uses an array of infrared sensors. It runs on windows, Mac \& Linux environment. It supports development in $\mathrm{C \#}, \mathrm{C}++$, Objective C, Java, Python, \& JavaScript. It contains API's for common gesture for rapid prototyping.

The Proposed system is based on the "Natural" human-robot interaction. Leap Motion is a computer hardware sensor device that supports hand and finger motions as input, analogous to a mouse, but requiring no hand contact or touching. Based on the sensor's coordinate system the sensor reports data such as speed and position of palm and fingers. All the information captured by the controller and then it fed to the algorithm. To obtain the mapping between the real arm and robotic arm, the current and previous information supplied by the controller is used. For enabling a more realistic imitation of the real hand, the arm's angular features such as roll, pitch, and yaw is considered for mapping procedure. The grasping and releasing operation performed by a robotic arm. The Proposed block diagram represented in Fig 3.

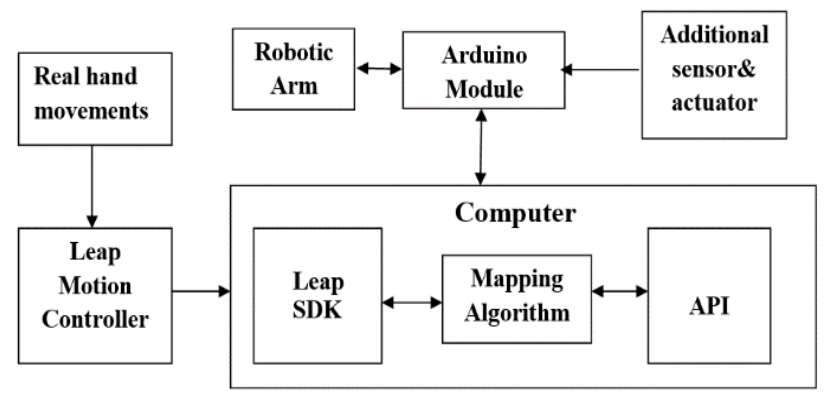

Fig. 3 Proposed Block Diagram

The system was developed in two stage. In the first stage of development, all information regarding the user's Palm Cartesian positions such as $\mathrm{X}, \mathrm{Y}$, and $\mathrm{Z} \&$ angular features such as roll, pitch, and yaw were retrieved from the Leap Motion Controller and fed to the mapping algorithm, implemented using the API and the Leap Motion Controller SDK files. In this stage, the number of hands and number of finger present in interaction box, are also detected.

In the second stage of development, first the Arduino Uno board is interfaced with leap motion and then fingers of the robotic arm were programmed to follow all grasp and release operations performed by the user. For developing applications for the Leap Motion device (usually to control robots), the Eclipse Integrated Development Environment is preferred. The Eclipse IDE was originally created for Java. After that, the hand's angular characteristics such as roll, pitch, and yaw angles were considered in the mapping procedure, enabling a more realistic imitation of the human arm.

To enhance the functionality of the system, an interface is established between the developed software and the Arduino Uno microcontroller [9]. Due to this any additional sensor, Actuator or display system can interface using Arduino like LEDs, pushbutton, and LCD display. 


\section{CONCLUSION}

In this paper, the various technologies used for Gesture Controlled Robotic Arm as well as Leap motion controller are specified. For Gesture Controlled system, the excellent features of Leap motion sensor lead to the development of a vigilant module. Kinect and Gyro-accelerometer sensors need to be replaced because of their high cost and less sensitivity. Microsoft Kinect sensors are previously used for Gesture Control, they are used for the first time in camera in which the subject is placed in front of the plane and Kinect generates the depth image of that image. Leap Motion Controller Having High Sensitivity, Enhanced Precision, and Compact size hence, it gives faster results than other gesture control sensor. The main objective of this study is to introduce a simple and straightforward robotic arm manipulation scheme, in order to enable the incorporation of robotic systems into the home environment.

\section{ACKNOWLEDGMENTS}

The authors would like to acknowledge the support of K.K.Wagh Institute of Engineering Education \& Research, Nasik, India for providing research facilities.

\section{REFERENCES}

[1] Lengare, P.S.; Rane, M.E., "Human hand tracking using MATLAB to control Arduino based robotic arm," in Pervasive Computing (ICPC), 2015 International Conference on, vol., no., pp.1-4, 8-10 Jan. 2015

[2] Allen, P.K.; Timcenko, A.; Yoshimi, B.; Michelman, P., "Automated tracking and grasping of a moving object with a robotic hand-eye system," in Robotics and
Automation, IEEE Transactions on, vol.9, no.2, pp.152165, Apr 1993

[3] Marin, G.; Dominio, F.; Zanuttigh, P., "Hand gesture recognition with leap motion and Kinect devices," in Image Processing (ICIP), 2014 IEEE International Conference on, vol., no., pp.1565-1569, 27-30 Oct. 2014

[4] Ching-Hua Chuan; Regina, E.; Guardino, C. "American Sign Language Recognition Using Leap Motion Sensor" Machine Learning and Applications (ICMLA), 2014 13th International Conference on Year: 2014

[5] Leap Motion - Mac and PC Motion Controller for Games, Design, and More. 2014. Available at http://www.leapmotion.com

[6] Ganesh Choudhary, B.; Chethan Ram, B.V., "Real-time robotic arm control using hand gestures," in HighPerformance Computing and Applications (ICHPCA), 2014 International Conference on, vol., no., pp.1-3, 2224 Dec. 2014

[7] Biswas, K.K.; Basu, S.K., "Gesture recognition using Microsoft Kinect ${ }^{\circledR}, "$ in Automation, Robotics, and Applications (ICARA), 2011 5th International Conference on, vol., no., pp.100-103, 6-8 Dec. 2011

[8] Bhuyan, A.I.; Mallick, T.C., "Gyro-accelerometer based control of a robotic Arm using AVR microcontroller," in Strategic Technology (IFOST), 2014 9th International Forum on, vol., no., pp.409-413, 21-23 Oct. 2014

[9] Arduino Uno Module Available at https://www.arduino.cc 